\title{
In Vitro and in Vivo Densitometric Analysis of DNA Content and Chromatin Texture in Nuclei of Tumor Cells under the Influence of a Nano composite and Magnetic Field
}

\author{
Natalia Boroday ${ }^{1}$, Vasily Chekhun ${ }^{2}$, Ekaterina Golubeva ${ }^{3}$ and Dmitry Klyushin ${ }^{4}$ \\ ${ }^{1,2}$ RE Kavetsky Institute of Experimental Pathology, Oncology and Radiobiology, \\ National Academy of Sciences of Ukraine, Kyiv, Ukraine \\ ${ }^{3,4}$ Taras Shevchenko National University of Kyiv, Kyiv, Ukraine
}

Correspondence should be addressed to: Dmitry Klyushin; dokmed5@gmail.com

Received date: 23 July 2014; Accepted date: 22 October 2014; published date: 23 August 2016

Copyright (c) 2016. Natalia Boroday, Vasily Chekhun, Ekaterina Golubeva and Dmitry Klyushin. Distributed under Creative Commons CC-BY 4.0

\begin{abstract}
DNA content and chromatin texture of interphase nuclei in MCF-7 and Guerin carcinoma cells that are sensitive or resistant to chemotherapeutic agents in the setting of the treatment with cisplatin, nanocomposite and static magnetic field have been analyzed. The results obtained indicate that chemotherapy agents and physical factors both in vitro and in vivo cause the increase in the DNA content. Tumor exposure to chemotherapy agents, nanocomposite and magnetic field causes the increase in the percentage of heterochromatin in nuclei of resistant tumor cells compared with sensitive ones, especially under the influence of combination of nanocomposite with static magnetic field. Probably it suggests the specific effects of combination of nanocomposite with magnetic field on cell chromatin depending on the changes in the strength of the DNA-histone interactions.
\end{abstract}

Keywords: Cancer, DNA content, Chromatin texture, Sensitive and resistant cell; MCF-7, Guerin carcinoma, Nanocomposite, Static magnetic field.

\section{Introduction}

In past years, considerable experimental information on the deterministic chromosome disposition at interphase and ordered patterns of chromatin packaging was accumulated indicating that the distribution of genetic material within the interphase nucleus is nonrandom (see Georgiev and Bakaev (1978), Grewal and Elgin (2002), Li et al. (2007)).

Cite this Article as: Natalia Boroday, Vasily Chekhun, Ekaterina Golubeva and Dmitry Klyushin (2016)," In Vitro and in Vivo Densitometric Analysis of DNA Content and Chromatin Texture in Nuclei of Tumor Cells under the Influence of a Nano composite and Magnetic Field ", Advances in Cancer Research \& Treatment, Vol. 2016 (2016), Article ID 706183, DOI: 10.5171/2016.706183 
There are two kinds of chromatin: heterochromatin and euchromatin. They are distinguished by genetic activity or, in other words, functional state of the cells, and differ by localization within nucleus, sensitivity to dissociating agents and nucleases, and the involvement in DNA conformational transitions and condensation mechanism (see $\mathrm{Li}$ et al. (2007), Kraevskii V. et al. (1992)). Heterochromatin is of significant importance in maintaining 3D-structure of an interphase nucleus and the basic properties and functions of genetic mechanisms (replication, transcription, recombination). Moreover, heterochromatin determines nonrandom pattern of interchromosomal exchanges offering the opportunities for the evolutional rearrangements of karyotype (see Li et al. (2007), Kumaran et al. (2008), Mazumder and Shivashankar (2007), Gordiyuk (2010), Kauzarides (2007)).

It was found that the morphology of the interphase chromatin changes significantly during the cell life cycle due to the complex processes of replication and transcription. However, normally these changes are limited within the physiological metabolic ranges. In various pathological conditions, interphase nucleus is affected significantly. The interphase chromatin is more sensitive to various endogenous and exogenous factors such as chemotherapeutic agents and magnetic fields than metaphase chromosomes. In a series of studies, it was shown that chemotherapeutic agents and magnetic fields cause compaction of chromatin (see Shkorbatov et. al. (2010), Skamrova (2012)).

The chromatin of inactive genes remains in the condensed form. Meanwhile, the active chromatin is decondensed. The decondensation of the chromatin allows for fast and free access of the transcription factors to DNA resulting in higher conformational mobility of DNA. The nucleosomes in the condensed chromatin are packaged more compactly limiting essentially the conformational changes in DNA. The nucleosomes of the active chromatin have decompacted, unfold structure with weakened DNA-protein interactions (Mazumder and Shivashankar (2007), Sacha et al. (2006), Lorch et al.
(2010)).

The basic proteins - histones (H1, H2A, $\mathrm{H} 2 \mathrm{~B}, \mathrm{H} 3$ and H4) and protamines - play the key role in the supporting of the highest levels of DNA organization (see Sacha et al. (2006), Lorch et al. (2010)). The histones are involved in regulation of activity of the genome in normal and tumor cells. Some investigators suppose that the histones inhibit genes expression, but others assert that histones induce topology changes in a region of chromatin in transition from inactive to active state (see Skamrova (2012), Felsenfeld and Groudine (2003), Peters et al. (2003) , Lim et al. (2010)).

Chromatin modifications induced by RNA play an important role in nucleosome decompaction (see Pezer (2008), Tomilin (2009), Patrushev and Minkevich (2008)). RNA is transcribed from the most regions of genome. When it forms the duplex structures as a result of transcription or extension by RNA-dependent RNApolymerase, the heterochromatin is being created (see Bondarenko et al. (2006), Kireeva (2002), Kloc and Martienssen (2008)). The mitotic phosphorylation of Ser-10 in histone $\mathrm{H} 3$ stimulates the transcription of heterochromatin and provides its reconstruction in the next cycle due to RNA interference (Kireeva (2002)). Hypothetically, the strengthening of heterochromatization is the result of genetic inactivation of the part of the genome. As a result, the information about elementary cell metabolic destructive processes is lost or the specific functions are terminated. In the latter case, the cell is alive but the pathological processes are developing (Lorch (2012)).

The modern cytometric methods of video microscopy and the methods for computeraided digital image analysis allow using the chromatin as a feature of the functional state of cells and their genetic mechanism affected by various factors including chemotherapeutic agents, nanocomposites and magnetic fields (Dudkin et al. (1999)). The changes of parameters of the chromatin distribution reflect the changes in the activating genes, and the quantitative content of DNA in cell nuclei may be indicative of the state of the genome. 
Thus, the purpose of our study is in vitro and in vivo analysis of DNA content and chromatin texture in interphase nuclei of tumor cells affected by nanocomposites and magnetic fields.

\section{Materials and Methods}

MCF-7 line of human breast cancer cells and the sublines resistant to doxorubicin (Dox) and cisplatin (CP) were used in the study. (The authors would like to thank Dr. Igor M. Todor, Department of Mechanisms of AntiTumor Therapy, RE Kavetsky Institute of Experimental Pathology, Oncology and Radiobiology of National Academy of Sciences of Ukraine, who provided the resistant strain.) The cells were cultured for 24 and 72 hours under the standard conditions with addition of the nanocomposite consisting of an iron nanocomplex in liposomal form. The iron concentration in the complex was $0.001 \%$.

In the second research series, the original MCF-7 cells and the cells of MCF-7/DDP subline resistant to cisplatin were cultured with addition of the cisplatin and magnetic fluid conjugated with the cisplatin at the dose corresponding to IC30. Cisplatin was added to the original MCF-7 cells at a concentration of $2 \mathrm{mcg} / \mathrm{ml}$. The concentration of cisplatin in the magnetic fluid was equal to $1.5 \mathrm{mcg} / \mathrm{ml}$, with $\mathrm{Fe}_{3} \mathrm{O}_{4}$ content of $24 \mathrm{mcg} / \mathrm{ml}$. Cisplatin was added to the cells of the resistant subline at a concentration of $4 \mathrm{mcg} / \mathrm{ml}$; the concentration of cisplatin in the magnetic fluid was equal to $3 \mathrm{mcg} / \mathrm{ml}$ with $\mathrm{Fe}_{3} \mathrm{O}_{4}$ content of $48 \mathrm{mcg} / \mathrm{ml}$. The cells were incubated with the studied substances for 24 hours under the standard conditions. Then, the cells of the original and resistant lines were exposed to the static field with induction of $156 \mathrm{mT}$ for 1 hour.

The histochemical study of cells was carried out in cytospin preparations fixed in $4 \%$ formaldehyde vapors for $3 \mathrm{~min}$. DNA content was determined in the nuclei stained by Feulgen according top the conventional technique.

Besides in vitro analysis, the effects of the nanocomposite were studied in Wistar rates (mass 130-150 g) with transplanted Guerin carcinoma (conventional strain or its cisplatin-resistant variant). In 9 days after transplantation, the animals with original Guerin carcinoma or cisplatin-resistant variant were divided into 5 groups and treated as follows: group I - control (no treatment); group II - intravenous injections of cisplatin "Ebeve" (Austria); group III - the same cisplatin injections as in group II followed by exposure of tumor to the static magnetic field (100-600 mT) following each injection for $30 \mathrm{~min}$; group IV - intravenous injections of nanocomposite (magnetic liquid with cisplatin) every other day, 4 injections in total at a dose of $1.2 \mathrm{mg}$ per $\mathrm{kg}$ of body mass and the dose of iron of $1 \mathrm{mg}$ per $\mathrm{kg}$ of body mass; group V - the same nanocomposite injections as in group IV followed by exposure of tumor to the static magnetic field (100-600 mT) following each injection for $30 \mathrm{~min}$. The rats were sacrificed according to the rules of bioethics for experimental studies on animals. The tumor material was fixed in $10 \%$ solution of neutral formalin. To prepare the histological specimens we used celloidin-paraffin embedding with further embedding in paraffin and preparation of sections 5-6 $\mu \mathrm{m}$ in thickness. The DNA content was estimated in nuclei of tumor cells stained by Feulgen.

The DNA cytometric analysis was carried out using the computer image analyzer consisted of Olympus microscope, Olympus C-5050 digital camera and computer. We measured the integral brightness of the stained nuclei $(\mathrm{zoom}=1000 \mathrm{x}$, wavelength $575 \mu \mathrm{m}$ ) that measures the total optical absorption in all pixels of nuclei image and represents the DNA content. We have developed an algorithm that was based on the analysis of distribution of absorption levels in a nuclei scanogram in order to determine condensed chromatin (CC) and decondensed chromatin (DC). We used the threshold of brightness $I_{t r}=I_{\min }+0.4 \times\left(I_{\max }\right.$ - $I_{\text {min }}$ ) to determine CC and DC. The pixel is corresponding to $\mathrm{CC}$ if its optical absorption falls within the range from Imin to $I_{\max }$. We considered that the pixel corresponds to $\mathrm{DCi}$ if this value exceeds the threshold. Counting total number of pixels corresponding to CC and DC and dividing it by the area of the nucleus (in pixels), we calculate the proportion of CC and DC in a nucleus. 
We determined the mean integral absorption of nuclei of small lymphocytes in smears of centrifugates of rat blood in order to determine the standard of ploidy. This standard corresponded to diploid $-2 \mathrm{n}$. The data on ploidy of tumor cells were obtained automatically: dividing the integral brightness of the nuclei of tumor cells by the half of cytological ploidy standard which was taken as a unit of measurement.

\section{Results and Discussion}

The DNA content in MCF-7 breast cancer cells resistant to doxorubicin and cisplatin was higher than in original MCF-7 culture: $152.4 \pm 6.02$ conventional unit (CU);

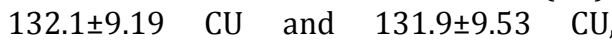
respectively (Tables 1 and 2).

Increase in DNA content in cisplatin- resistant cells in comparison with sensitive ones can be explained by the fact that according to literature data the development of resistance is accompanied by the changes of the karyotype profile of these cells, the chromosomal aberrations driven by genes amplification and the accelerated reparation of DNA. According to literature data, the resistance may be associated with the activation of oncogenes (Svirnovsky and Pasyukov (2007)).

The changes of the chromatin texture demonstrate a trend towards intensification of heterochromatization in nuclei of resistant cells. This can be explained by the oxidative stress caused by the direct interaction with DNA and penetration of ferromagnetic through the cell membrane (Alkhateeb and Connor (2010)).

Table 1: DNA content and proportion of condensed (CC) and decondensed (DC) chromatin in nuclei of original MCF-7 cells exposed to nanocomposite

\begin{tabular}{|c|c|c|c|}
\hline Factors, CU & \multicolumn{3}{|c|}{ Time of nanocomposite exposure } \\
\hline & $0 \mathrm{~h}$ & $24 \mathrm{~h}$ & $72 \mathrm{~h}$ \\
\hline DNA & $131.9 \pm 9.53$ & $135.1 \pm 4.94$ & $143.1 \pm 6.02$ \\
& $71.1-168.9$ & $98.5-178.4$ & $19.8-178.5$ \\
\hline CC & $0.41 \pm 0.07$ & $0.30 \pm 0.07$ & $0.32 \pm 0.07$ \\
\hline DC & $0.59 \pm 0.07$ & $0.70 \pm 0.07$ & $0.68 \pm 0.07$ \\
\hline
\end{tabular}

Table 2: DNA content and proportion of condensed (CC) and decondensed (DC) chromatin in nuclei of cells in doxorubicin and cisplatin resistant MCF-7 sublines exposed to nanocomposite

\begin{tabular}{|c|c|c|c|c|c|c|}
\hline $\begin{array}{c}\text { Factors, } \\
\text { CU }\end{array}$ & \multicolumn{3}{|c|}{$\begin{array}{c}\text { Dox resistant MCF-7 } \\
\text { Time of nanocomposite exposure }\end{array}$} & \multicolumn{2}{c|}{$\begin{array}{c}\text { Cisplatin resistant MCF-7 } \\
\text { Time of nanocomposite action }\end{array}$} \\
\hline & $0 \mathrm{~h}$ & $24 \mathrm{~h}$ & $72 \mathrm{~h}$ & $0 \mathrm{~h}$ & $24 \mathrm{~h}$ & $72 \mathrm{~h}$ \\
\hline DNA & $152.4 \pm 6.02$ & $142.7 \pm 6.88$ & $152.4 \pm 6.01$ & $132.1 \pm 9.19$ & $138.4 \pm 6.88$ & $147.1 \pm 4.31$ \\
& $122.4-$ & $97.2-171.9$ & $126.0-$ & $84.8-173.0$ & $97.2-$ & $114.1-175.6$ \\
& 175.7 & & 177.3 & & 171.9 & \\
\hline CC & $0.34 \pm 0.07$ & $0.31 \pm 0.07$ & $0.36 \pm 0.07$ & $0.30 \pm 0.07$ & $0.31 \pm 0.07$ & $0.33 \pm 0.07$ \\
\hline DC & $0.66 \pm 0.07$ & $0.69 \pm 0.07$ & $0.64 \pm 0.07$ & $0.70 \pm 0.07$ & $0.69 \pm 0.07$ & $0.67 \pm 0.08$ \\
\hline
\end{tabular}

As a result, the cross-linking between DNA thymine and tyrosine of histone activates. In contrast, decondensed chromatin increases in the nuclei of original MCF-7 cells exposed to nanocomposite. The chromatin decondensation is accompanied by the activation of transcription in cells ( $\mathrm{Li}$ et al. (2007)). The activation of chromatin is based on weakening of DNA-protein interactions with decreasing the shielding effect of histones (Grewal and Elgin (2002)). It is well-known that histone amino groups interact with phosphate groups of DNA preventing the interaction of ligands with DNA within deoxyribonucleic acid complex. The higher level of the histone 
acetylation is characteristic of the active chromatin. This causes the neutralization of their amino groups and weakening of their linkage with DNA. The increase in decondensed chromatin proportion due to partial loss or modification of histone $\mathrm{H} 1$ is facilitated by DNA alkylation with cisplatin. Alkylated DNA activates linkage with several histones, primarily, H1. Also, the chromatin decondensation provides for fast and free access of the transcription factors to DNA ensuring higher conformational mobility of DNA (Chekhun et al. (2007)).

Following 72 hours of incubation with ferromagnetic-contained nanocomposite, DNA content in the original MCF-7 cells increased from $131.9 \pm 9.53 \mathrm{CU}$ to 143.1 $\pm 6.02 \mathrm{CU}$ (Table 2). In this setting, DNA content in the cisplatin-resistant MCF7 cells also increased while in Dox-resistant MCF-7 cells the treatment with nanocomposite did not affect DNA content (Table 2). This may be explained by the fact that doxorubicin can block $\mathrm{G}_{2}$ phase of mitotic cycle.
The effects of cisplatin, magnetic fluid, and nanocomposite of the DNA content and chromatin redistribution in cisplatin sensitive and resistant cells are presented in Table 3. The DNA content in the resistant cells was higher as compared to original MCF-7 cells: 127.2 and $121.9 \mathrm{cu}$, respectively.

The DNA content increased both in original MCF-7 cells and cells of resistant sublines exposed to cisplatin, magnetic fluid and nanocomposite. It is known that cisplatin delays the cell cycle in S-phase and arrest the cells in G2/M-phase (Vaisman et al. (1997)). The arrest of cell cycle in G2-phase allows for repairing damaged DNA preventing the emergence of mutant and aneuploid cells. It is well-known that the reparation in this process is a stage of excisional reparation which means the breaks repairing in course of DNA replication involving DNA polymerase (Fhon (2008)).

Table 3: DNA content and proportion of condensed (CC) and decondensed (DC) chromatin in nuclei of MCF-7 and MCF-5/DDP cells exposed to cisplatin, magnetic Fluid and nanocomposite

\begin{tabular}{|c|c|c|c|c|c|c|}
\hline \multirow[t]{2}{*}{ Treatment } & \multicolumn{3}{|c|}{ MCF-7 } & \multicolumn{3}{|c|}{ MCF-7/DDP } \\
\hline & DNA. cu & CC. cu & DC. cu & DNA. cu & CC. cu & DC. cu \\
\hline Control & $\mid \begin{array}{l}121.9 \pm 11.1 \\
80.4-160.2\end{array}$ & $0.26 \pm 0.06$ & $0.74 \pm 0.08$ & $\begin{array}{c}127.2 \pm 9.2 \\
90.0-155.5\end{array}$ & $0.24 \pm 0.06$ & $0.75 \pm 0.08$ \\
\hline Cisplatin & $\mid \begin{array}{l}123.4 \pm 11.1 \\
80.4-160.2\end{array}$ & $0.25 \pm 0.07$ & $0.75 \pm 0.07$ & $\begin{array}{c}129.2 \pm 9.2 \\
90.0-155.5\end{array}$ & $0.29 \pm 0.06$ & $0.72 \pm 0.08$ \\
\hline Magnetic fluid & $\begin{array}{c}125.1 \pm 11.6 \\
73.4-123.3\end{array}$ & $0.26 \pm 0.06$ & $0.75 \pm 0.08$ & $\begin{array}{c}128.8 \pm 8.1 \\
88.1-163.3\end{array}$ & $0.30 \pm 0.06$ & $0.71 \pm 0.07$ \\
\hline Nanocomposite & $\mid \begin{array}{c}125.3 \pm 7.8 \\
77.8-160.6\end{array}$ & $0.25 \pm 0.07$ & $0.75 \pm 0.07$ & $\begin{array}{c}129.8 \pm 12.3 \\
88.8-163.3\end{array}$ & $0.37 \pm 0.07$ & $0.63 \pm 0.07$ \\
\hline
\end{tabular}

The percentage of decondensed chromatin in the nuclei of original MCF-7 cells exposed to cisplatin, magnetic fluid and nanocomposite increased (Table 3). Primarily, this can be explained by the different content of condensed and decondensed chromatin in nuclei depending on the phase of cell cycle (Kloc and
Martienssen (2008)). It is known that early at S-phase the condensed chromatin disappears with transition into diffusible form. Then, at the late G2-stage the chromatin condensed again that is associated with formation of prophase chromosomes. Since cisplatin causes the delay in S-phase with G2/M arrest (see 
Vaisman et al. (1997), Fhon (2008)), we observed the decrease in condensed chromatin area (due to DNA synthesis), while post-synthetically, the number of zones of condensed chromatin increased.

On the contrary, in nuclei of cisplatin resistant cells exposed to cisplatin, magnetic fluid and nanocomposite the percentage of condensed chromatin increased. There are many data on protective mechanisms in cells on genetic and cytological levels as a response to endogenous and exogenous factors (Englevsky and Brazhnikova (2010)). Condensed state of structural chromatin is of particular importance in this aspect. As far as structural chromatin resides at internal side of nuclear envelope, it protects the critically important regions of chromosomes with genes to be transcribed.

Recently it has been suggested that the heterochromatin is partially destroyed and restored at every mitosis with the involvement of RNA interference. This scheme is considered as a model of reconstruction of modified histones that provides heterochromatin (Fhon (2008)) and even as a general model of epigenetic inheritance in eukaryotes driven by RNAinterference (Kloc and Martienssen (2008)). The key part in mitosis is the phosphorylation of Ser-10 (HP1) and activation of transcription in G1 and early Sphase. The transcripts of heterochromatin are accumulated in S-phase and transformed to specific siRNA-complexes (RITS-complexes - RNA Induced Transcription Silencing) inducing additional methylation of Lys-9 in histone H3 (after replication) and binding with Swi 6 protein. Thus, the heterochromatin is completely reconstructed in each new cycle.

The exposure to the magnetic field of both original and resistant MCF-7 cells cultured in the presence of cisplatin, magnetic liquid and nanocomposite resulted in the increased proportion of condensed chromatin and the decreased proportion of decondensed chromatin. This is in accordance with literature data that a magnetic field promotes compaction of chromatin (Kraevskii et al. (1992), Kumaran et al. (2008), Sarimov et al. (2011)). It is known that negatively charged DNA and positively charged histones in nucleosomes interact electrostatically. This interaction affects the chromatin state and the changes if magnetic field induces currents in the body (see Sarimov R., Alipov and Belyaev (2011), Luger et. al. (1997), Hilder and Maclean (1974), Korolev et. al. (2012)). There are various views on the mechanisms of interactions between the electromagnetic fields and the living organisms. The main hypothesis is based on polarization of bound charges, orientation of constant dipoles and movement of free histones (McKinlay and Bernhardt (2003)). At the nucleosomal level this effect can be manifested as redistribution of the charges of nucleosomal components leading to the strengthening of the histon-DNA interactions, in other words, chromatin condensation.

In in vivo experiments, the DNA content in nuclei of Guerin carcinoma cells of the conventional cisplatin-sensitive strain was higher in comparison to the resistant type of tumor: $67.3 \pm 17.8$ C.U. and $65.6 \pm 19.4$ C.U. respectively (Table 4).

The exposure to the various factors understudy did not affect DNA content in nuclei of tumor cells of both sensitive and resistant carcinoma. The percentage of condensed chromatin in cells of cisplatin sensitive Guerin adenocarcinoma in control animals was lower in comparison to nuclei of cells of cisplatin resistant Guerin adenocarcinoma. This value decreased in cisplatin sensitive tumors under the influence of cisplatin, nanocomposite and cisplatin, and nanocomposite, cisplatin and magnetic field while in cells of cisplatin resistant Guerin carcinoma the factors stated above resulted in the increasing proportion of the condensed chromatin. Therefore, we observed the trend of increasing condensed chromatin content in nuclei of cisplatin resistant tumors under the influence of various factors. Under the same conditions, the decondensed chromatin in nuclei of cells of cisplatin sensitive line of human breast cancer increased.

Also, we studied the imbalance of DNA (aneuploidy) in nuclei of tumor cells in tumor-bearing animals exposed to cisplatin, nanocomposite and cisplatin, cisplatin and 
magnetic field and combination of the factors above. The densitometric analysis of specimens has demonstrated that the ploidy in tumor cells of sensitive Guerin adenocarcinoma differed from that in cells of resistant adenocarcinoma (Figure 1).

In $60-68 \%$ of cells of cisplatin sensitive Guerin adenocarcinoma, the ploidy amounted to $3 \mathrm{n}$ and the percentage of cells with diploid set of chromosomes varied from 20 to $34 \%$. Meantime, the modal class of the resistant tumor cells contained mostly diploid cells with $2 \mathrm{n}$ ploidy (63\%). The modal class of cells in resistant tumor affected by cisplatin corresponded to the level of diploid cells (62\%), and in sensitive tumors the number of cells with $3 \mathrm{n}$ ploidy increased to $44 \%$, and the proportion of diploid cells was $34 \%$.

It is worthy of note that sensitive tumors contained more tetraploid cells (4n) composing $16 \%$ of total number of tumor cells under the influence of cisplatin and $23.6 \%$ under the influence of nanocomposite compared with resistance tumors. In the group of animals with the resistant Guerin carcinoma exposed to cisplatin, the modal class contained diploid cells $(58 \%)$, and in the group of sensitive tumors the modal class contained triploid cells $(43 \%)$. The increase in tetraploid cells content is an evidence of the increasing aneuploidy. It should be noted wider scattering of the cells with respect to ploidy in the sensitive tumors - from $1 \mathrm{n}$ до $4 \mathrm{n}$, while the ploidy in the resistant tumors was $2 \mathrm{n}$ or $3 \mathrm{n}$. The ploidy of tumor cells in animals with cisplatin sensitive adenocarcinoma that received cisplatin and was affected by magnetic field was $2 \mathrm{n}$ and $3 \mathrm{n}$. The proportions of such cells were $24 \%$ and $44 \%$ respectively with modal class $3 \mathrm{n}$. The resistant tumors contained $42 \%$ and $43 \%$ of the diploid and tetraploid cells, respectively. The ploidy of cells in resistant tumors in animals receiving the nanocomposite and affected by magnetic field was $2 \mathrm{n}$ and $3 \mathrm{n}-56 \%$ and $34 \%$, respectively with the modal class of $2 n$. The cells with $3 \mathrm{n}$ ploidy predominated in the sensitive tumors (45\%) where the percentage of the cells with $2 \mathrm{n}$ and $4 \mathrm{n}$ ploidy was $22 \%$ and $21 \%$, respectively. The modal class in resistant tumors under the influence of nanocomposite and magnetic field contained mostly diploid cells (62\%), while the modal class in sensitive adenocarcinomas contained both diploid (50\%) and tetraploid cells (38\%). Our data are in accordance with the data of other investigations demonstrating the increasing aneuploidy index. This proves the imbalance of the DNA exchange under the influence of electromagnetic fields and ferromagnets (see Ivanov et al. (2010), Mashevich et al. (2003), Ivancsits et al. (2002)). 


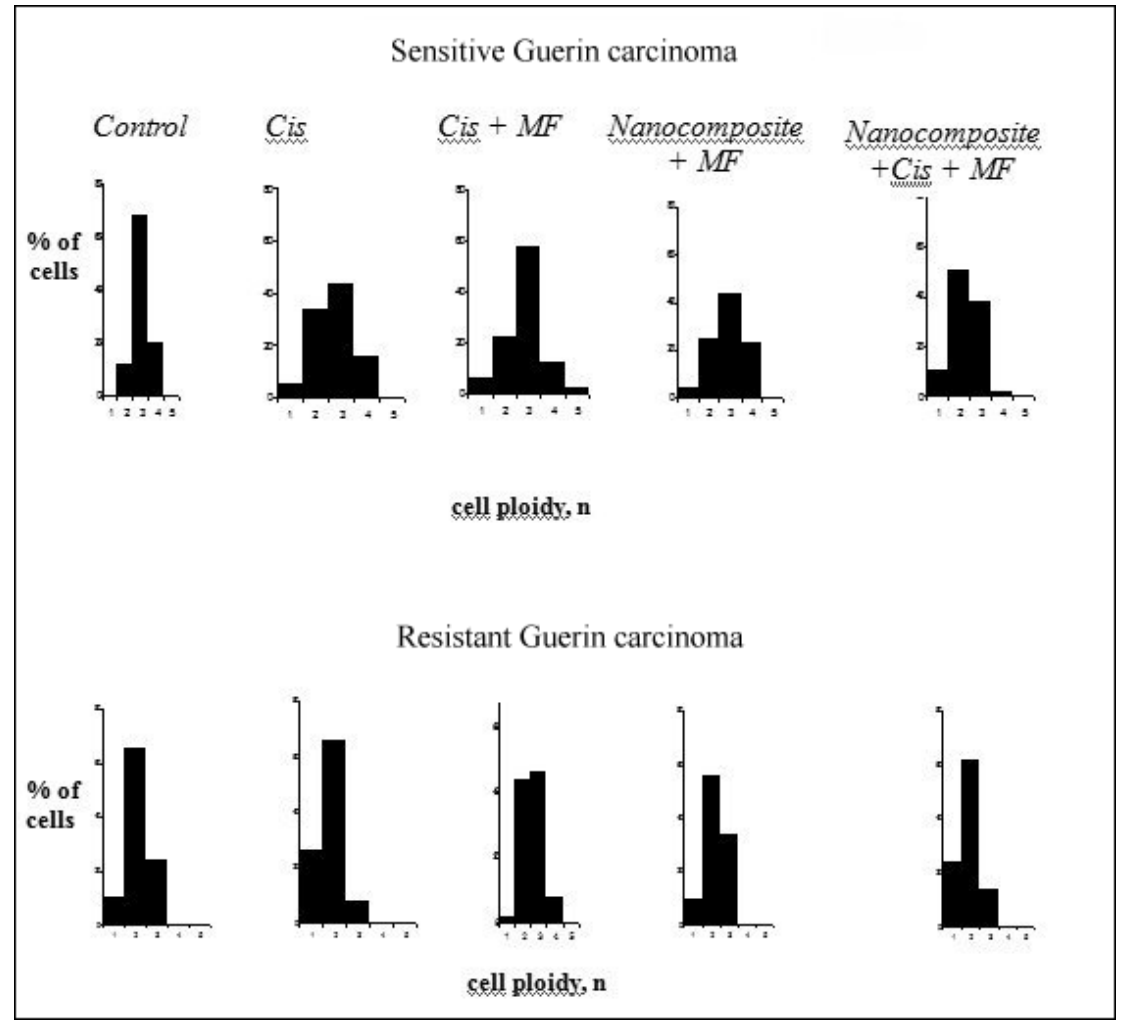

Figure 1: DNA content in nuclei of tumor cells of cisplatin sensitive and resistant Guerin carcinoma under the influence of various factors

Thus, the most explicit abnormalities in genetic mechanisms of cells were detected in tumors of the animals with sensitive Guerin carcinoma under the combined influence of a nanocomposite and magnetic field. Such abnormalities were manifested by higher proportion of tetraploid cells and the shift of histogram towards the right that points to the change of karyotype in these cells. Hypothetically, such aberrations of cell ploidy may be indicative of 1) conformation changes in chromatin (changes of the DNAhistone interactions); 2) damage of the nucleus membrane structure that can cause formation of different ploidy classes; and 3) abnormalities of formation of cleavage spindle.

Table 4: DNA content and chromatin distribution in nuclei of cisplatin sensitive Guerin carcinoma cells under the influence of various factors

\begin{tabular}{|c|c|c|c|c|c|}
\hline Factors & Control & Cisplatin & $\begin{array}{c}\text { Cisplatin + } \\
\text { magnetic field }\end{array}$ & $\begin{array}{c}\text { Cisplatin + } \\
\text { magnetic fluid } \\
\text { (nanocomposite) }\end{array}$ & $\begin{array}{c}\text { Cisplatin + } \\
\text { magnetic } \\
\text { fluid + } \\
\text { magnetic } \\
\text { field }\end{array}$ \\
\hline \multicolumn{5}{|c|}{ Cisplatin sensitive Guerin carcinoma } \\
$\begin{array}{c}\text { DNA } \\
\text { content . } \\
\text { c.u. }\end{array}$ & $67.3 \pm 17.8$ & $68.6 \pm 18.8$ & $66.98 \pm 18.4$ & $68.2 \pm 19.7$ & $67.6 \pm 17.9$ \\
\hline
\end{tabular}

Natalia Boroday, Vasily Chekhun, Ekaterina Golubeva and Dmitry Klyushin (2016), Advances in Cancer Research \& Treatment, DOI: 10.5171/2016.706183 


\begin{tabular}{|c|c|c|c|c|c|}
$\begin{array}{c}\text { Range of } \\
\text { DNA } \\
\text { content }\end{array}$ & $25.6-133.9$ & $18.9-130.1$ & $15.3-123.5$ & $19.8-154.1$ & $11.3-133.4$ \\
\hline CC & $0.37 \pm 0.07$ & $0.34 \pm 0.07$ & $0.34 \pm 0.07$ & $0.34 \pm 0.07$ & $0.34 \pm 0.07$ \\
\hline DC & $0.63 \pm 0.07$ & $0.67 \pm 0.07$ & $0.66 \pm 0.07$ & $0.66 \pm 0.07$ & $0.66 \pm 0.07$ \\
\hline \multicolumn{5}{|c|}{ Cisplatin resistant Guerin carcinoma } \\
\hline $\begin{array}{c}\text { DNA } \\
\text { content. } \\
\text { c.u. }\end{array}$ & $65.6 \pm 19.4$ & $61.6 \pm 18.6$ & $66.5 \pm 18.2$ & $64.1 \pm 17.7$ & $65.9 \pm 23.1$ \\
\hline $\begin{array}{c}\text { Range of } \\
\text { DNA } \\
\text { content }\end{array}$ & $10.2-123.6$ & $8.4-122.5$ & $11.3-127.5$ & $9.7-120.2$ & $12.0-121.4$ \\
\hline CC & $0.45 \pm 0.07$ & $0.47 \pm 0.06$ & $0.47 \pm 0.06$ & $0.47 \pm 0.07$ & $0.47 \pm 0.07$ \\
\hline DC & $0.55 \pm 0.07$ & $0.53 \pm 0.06$ & $0.53 \pm 0.07$ & $0.53 \pm 0.07$ & $0.53 \pm 0.07$ \\
\hline
\end{tabular}

\section{Conclusions}

The results of our research demonstrate that the DNA content increases in nuclei of both sensitive and resistant tumor cells exposed to chemotherapeutic agents and physical factors in vitro and in vivo. We have shown that chemotherapy agents, nanocomposite and magnetic field increased the percentage of heterochromatin in the resistant cells as compared with the sensitive cells. This

\section{References}

1. Alkhateeb A. and Connor J. (2010) "Nuclear ferritin: A new role for ferritin in cell biology," Biochem. Biophys Acta, 1800(8): 793-97.

2. Bondarenko R., Steele L., Ujvrari A. et al. (2006) "Nucleosomes can form a polar barrier to transcript elongation by RNA polymerase II," Mol Cell, 24: 469-79.

3. Chekhun V., Lukyanova N. and Kovalchuk 0. (2007) "Epigenetic profiling of multidrug-resistant human MCF-7 breast adenocarcinoma cells reveals novel hyperand hypomethylated targets," Mol Cancer Ther., 6(3): 1089-98.

4. Dudkin K., Mironov S., Diuzhikova N., Lopatina N. (1999) "An information system for automated cytogenetic image processing: analysis of interphase chromatin of nerve cells of embryonic brain of rats with varying nervous system excitability," Cytology, 41(3-4): 237-49. increase becomes even more explicit under the exposure to magnetic field. The mechanism of formation of ploidy classes in tumor cells of cisplatin resistant Guerin carcinoma is impaired or absent. The data suggest the specific mechanisms of combined influence of nanocomposite and magnetic field on cell chromatin depending on the changes of the strength of the DNAhistone interactions.

\section{Russian.}

5. Englevsky N. and Brazhnikova E. (2010) "Defensive functions of condensed chromatin against influence of endo- and exogenic factors on cell nucleus: survey," Proceedings of the International conference "Problems and perspectives of modern medicine, biology and ecology". Aundamental sciences and practics, 1(2): 7-10.

6. Felsenfeld G. and Groudine M. (2003) "Controlling the double helix," Natrure, 421: 448-53.

7. Fhon G.. (2008) "Histone modifications: cycling with chromosomal replication," Curr. Biol, 18: 380-382.

8. Georgiev G.. and Bakaev V. (1978) "Three levels of structural organization of the eukaryotic chromosomes," Mol Biol, 12 (6): 1205-30. Russian.

9. Gordiyuk V. (2010) "Genetic and epigenetic changes in malignant cells of tumors of urogenital organs," Biopolimers and Cell, 26 (6): 450-457. Russian.

10. Grewal S. and Elgin S. (2002) 
"Heterochromatin: new possibilities for the inheritance of structure," Current Opinion in Genetics \& Development, N 12: 178-187.

11. Grewal S. and Elgin S. (2002) "Heterochromatin: new possibilities for the inheritance of structure," Current Opinion in Genetics \& Development, 12:178-87.

12. Hilder V. and Maclean N. (1974) "Studies on the template activity of "isolated" Xenopus erythrocyte nuclei," J Cell Sci., 16: 133-142.

13. Ivancsits S., Diem E., Pilger A. et al. (2002) "Induction of DNA stand breaks by intermittent exposure to extremely-cowfrequency electromagnetic fields in human diploid fibroblusts," Mutant Res., 529 (1-2): 1-13.

14. Ivanov S.D., Nikitina V.N., Lamshanov V.A. et al. (2010) "The influence of ferromagnetic screening from natural electromagnetic fields on the hematological and toxicogenomic indexes in animals," Radiats. Biol Radioecol, 50(2): 195-200.

15. Kauzarides T. (2007) "Chromatin modifications and their function," Cell, 128 (4): 693-705.

16. Kireeva M., Walter W., Tchernajenko V., Bondarenko V. et al. (2002) "Nucleosome remodeling induced by RNA polymerase II: Coss of the H2A/H2B dimer during transcription," Mol Cell, 9: 541-52.

17. Kloc A. and Martienssen R. (2008) "RNAi, heterochiomatin and the cell cycle," Frens. Genet., 6: 793-97.

18. Korolev N., Fan Y., Lyubartsev A. et. al. (2012) "Modelling chromatin structure and dynamics: status and prospects," Current opinion in structural biology, 22(2): 151159.

19. Kraevskii V., Mikhailov V. and Razin S. (1992) "Spatial organization and conformational mobility of DNA complexed with nucleoproteins," Mol Biol, 26(4):74556. Russian.

20. Kumaran R., Thakar R. and Spector D. (2008) "Chromatin dynamics and gene positioning," Cell, 132: 929-934.

21. Li B., Carey M. and Workman J.L. (2007) "The role of chromatin during transcription," Cell, 128: 707-719.
22. Lim S., Metzger E., Schule R., Kirfel J. et al. (2010) "Epigenetic regulation of cancer grouth by histone demethylases", Lut. J. of Cancer, 127 (9): 1991-98.

23. Lorch J., Maier-Davis B., and Konnberg R.D. (2010) "Mechanism of chromatin remodeling," PNAS, 107 (8): 3458-62.

24. Luger K., Mader A., Richmond R. et. al. (1997) "Crystal structure of nucleosome core particle at 2.8 A resolution," Nature, 389: 251-260.

25. Mashevich M., Folkman D., Kesar A. et al. (2003) "Exposure of human peripheral blood lymphocytes to electromagnetic fields associated with cellular phones leads to chromosomal instability," Bioelectromagnetics, 24 (2): 82-90.

26. Mazumder A. and Shivashankar G.V. (2007) "Gold-nanoparticle-assisted laser Perturbation of chromatin assembly reveals unusual aspects of nuclear architecture within living cells," Biophysical Journal, 93: 2209-2216.

27. McKinlay A., Bernhardt J. (2003) "ICNIRP - basic for EMF exposure guideline," $3^{\text {rd }}$ International EMF Seminar in China: Electromagnetic fields and biological effects, 63-67.

28. Patrushev L. and Minkevich I. (2008) "The problem of the eukaryotic genome size," Biochemistry, 73(13): 1519-52.

29. Peters A., Kubicek S. and Mechtler K. (2003) "Partitioning and plasticity of repressive hystone methylation states in mammalian chromatin," Mol Cell., 12: 157789.

30. Pezer Z. (2008) "Role of non-coding RNA and heterochromatin in aneuploidy and cancer," Cancer biology, 18: 123-130.

31. Sacha A., Wittmyger J. and Cairns B.R. (2006) "Chromatin remodeling: The industrial revolution on DNA around histones," Nat. Rev. Mol Cell. Biol., 7 (6): 437-447.

32. Sarimov R., Alipov E. and Belyaev I. (2011) "Fifty hertz fields individually affect chromatin conformation in human lymphocytes: dependence on amplitude, 
temperature, and initial chromatin state," Bioelectromagnetics, 32 (7): 570-579.

33. Shemarova I. (2008) "Posttranslation regulation of programmable cell events in unicellular eukaryotes," Cytology, 50 (80): 663-70. Russian.

34. Shkorbatov Y., Rudneva L., Pasiuga V. et. al. (2010) "Electromagnetic field effects on Artemia hatching and chromatin structure," Central Eur. J. of Biology, 5(6): 785-90.

35. Skamrova G., Evstigneev M., Trushkin A., Shkorbatov Yu. (2012) "Influence of electrical and magnetic components of electromagnetic field on the permeability of membranes and chromatin state in nuclei of cells of human buccal epithelium," Proceedings of of Taurida National V.I. 39.
Vernadsky University. Series of Biology and Chemstry, 25 (64): 187-195.

36. Svirnovsky A. and Pasyukov V. (2007) "Moluculas basis of phenomena of chemoand radioresistancy in tumor processes," Medical news, 11: 7-19. Russian.

37. Tomilin N. (2009) "Inheritance of RNAdirected epigenetic modifications of chromatin," Cytology, 51 (4): 291-296. Russian.

38. Vaisman A., Varchenko M., Suid L. et al. (1997) "Cell cycle changes associated with formation of Pt-DNA-adductsin human ovarion carcinoma cells with different cisplatin sensitivity," Cytometry, 27(1): 5662. 